\title{
Forceful biomarkers
}

The physical properties of living tissues are a compelling source of biomarkers of health and disease.

B iomarkers come in all sizes, shapes and colours. Step counts are a biomarker for physical activity, blood pressure is a clinical biomarker for hypertension (and a risk factor for stroke), and haemoglobin concentration for anaemia. Most biomarkers of disease - such as the concentration of chloride in sweat for cystic fibrosis, serum levels of the prostate-specific antigen for the prognosis of prostate cancer and mutations in the $B R C A$ genes for breast-cancer risk - are biochemical, cellular or genomic. This is a direct result of staggering advances in technologies for biosensing, single-cell analyses and genomics. In comparison, the physical properties of ensembles of cells and the extracellular matrix making up living tissues - that is, the properties that affect their morphology, structure and mechanical properties - have been a much less prolific source of specific biomarkers.

Many physical diseases have tissue-level hallmarks, and more of such microscopicto-macroscopic biomarkers (rather than of the molecular or cellular kind) could aid the understanding of disease risk, or help with diagnosis, prognosis or patient monitoring. For instance, carcinomas malignancies of epithelial tissue that account for more than $80 \%$ of all cases of cancer - cause, at an early stage, morphological changes at the tissue level. Arteriosclerosis (and also ageing) cause arteries to stiffen. And respiratory-tract infections lead to inflammation-driven changes in the physical properties of epithelial tissue in the airways. Also, white-light imaging, which is widely available in clinical centres, can detect morphological abnormalities (such as irregular vascular patterns) on the skin, and thus the potential presence of lesions; yet non-invasive biomarkers that predict the type and prognosis of a lesion are more difficult to come by. In fact, for epithelial tissues (not only the skin, but also the epithelium covering the airways, the digestive tract and the urinary system), only some tissue-level properties can be measured non-invasively or minimally invasively via dermoscopy and endoscopy. Instead, for non-epithelial solid tissues, if tissue biopsies are available a plethora of techniques can be used to analyse the morphology, structure and mechanical properties of the explanted tissue (in addition to a myriad of cellular, molecular, and genomic and transcriptomic
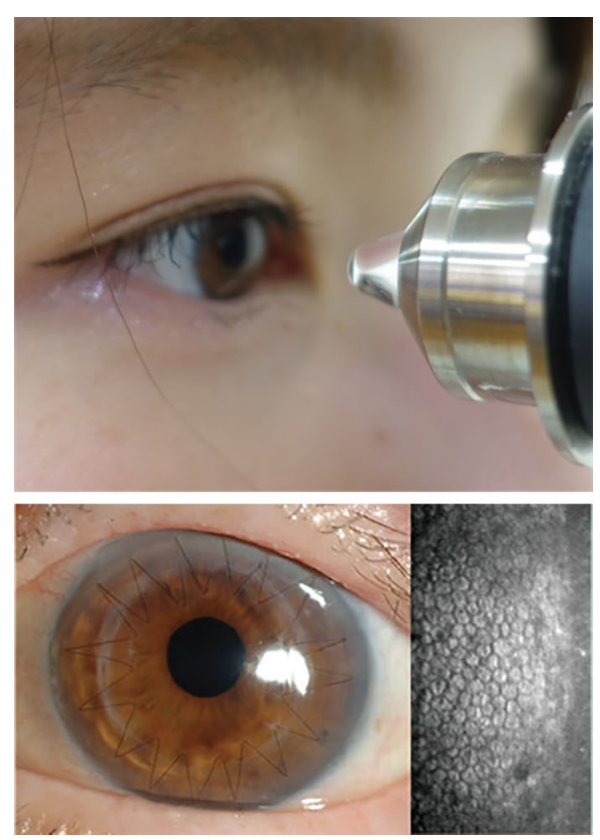

Figure adapted from. Yamamoto, A. et al. Nat. Biomed. Eng. https://doi.org/10.1038/s41551019-0429-9 (2019), Springer Nature Ltd.

analyses). When biopsied tissue is not available, comparably limited tissuelevel characteristics can be measured radiologically, such as the local density and morphology of blood vessels via magnetic resonance, the morphology and density of abnormal masses by using magnetic resonance or computed tomography, and the stiffness of tissue via ultrasound elastography.

Increased tissue stiffness is a wellknown hallmark of fibrotic tumours (and hence palpation of the breasts and of the testes are a recommended screening method), yet by itself this physical biomarker is not a strong predictor of malignancy; in fact, the underlying molecular and cellular links of tissue stiffness and tumorigenicity remain to be fully understood, as exemplified by an Article, by Ovijit Chaudhuri and colleagues included in in this issue, showing that changes in the stiffness of the extracellular matrix of mammary epithelium drive malignant phenotypes in the cells via changes in the accessibility of chromatin. In addition to changes in stiffness, tumours exhibit other abnormal mechanical properties, such as solid stress and stored elastic energy, which only recently have been quantitatively mapped in excised human tissues. And as shown earlier this year, for some types of brain tumour, solid stress is also a biomarker of the neurological dysfunction caused by the tumour's compression of adjacent brain tissue, which then experiences reduced blood perfusion (as seen with magnetic resonance imaging in patients).

This issue includes an exemplary discovery of a new tissue-level biomarker for the health status of corneal endothelium: a measure of the average 'spring-like' force that a cell in the endothelial monolayer experiences as a consequence of its interaction with the cell's immediate neighbours. The balance of forces that the cells experience and exert affect how the cells in the monolayer arrange themselves and thus their collective degree of order. This can be modelled by using basic concepts in statistical mechanics, as shown by Motomu Tanaka, Morio Ueno and colleagues in an Article. In particular, the researchers measured such a spring-like force by calculating the second derivative around the minimum value in the average force between pairs of cells as a function of their distance (known as the potential of mean force in statistical physics), which they obtained by taking specular microscopy images of patient corneas (pictured), and then measuring the average distribution of cells around every cell in the imaged endothelium (the so-called radial distribution function, the logarithm of which is directly related to the potential of mean force). Measurements of how objects in a thermal system organize themselves, and the derivation of the effective interactions between each pair of objects, are routine in colloidal physics. The researchers' insight that corneal epithelial cells physically behave, at the tissue level, just like packed colloidal particles, led them to the discovery of a biomarker that turned out to be useful in predicting, non-invasively, the prognosis of patients with transplanted corneas, as well as the quality of the cells when expanded and cultured as a confluent monolayer on a dish prior to implantation in patients. As noted by Gary Peh and colleagues in an accompanying News \& Views, 
the biomarker could help refine cell-culture and cell-expansion protocols.

The need for such physical biomarkers is forceful, in particular when in combination with the typically more specific and sensitive biochemical, molecular and cellular biomarkers. By leveraging transdisciplinary interactions between researchers who study the biology of disease, clinicians experiencing the need for and limitations of current biomarkers, and physicists and materials scientists working in, for example, colloidal physics, tissue biomechanics and medical imaging, more tissue-level biomarkers ought to be discovered.

Published online: 6 December 2019 https://doi.org/10.1038/s41551-019-0496-y 